\title{
Searching the Parameters of Dark Matter Halos on the Basis of Dwarf Galaxies' Dynamics
}

\author{
L. M. Chechin ${ }^{1,2}$, T. K. Konysbayev² \\ ${ }^{1}$ V.G. Fessenkov Astrophysical Institute, Almaty, Kazakhstan \\ ${ }^{2}$ Al-Farabi Kazakh National University, Almaty, Kazakhstan \\ Email: chechin-Im@mail.ru, talgar_777@mail.ru
}

Received 11 March 2016; accepted 24 May 2016; published 27 May 2016

Copyright (C) 2016 by authors and Scientific Research Publishing Inc.

This work is licensed under the Creative Commons Attribution International License (CC BY). http://creativecommons.org/licenses/by/4.0/

c) (i) Open Access

\section{Abstract}

Article devoted to searching the parameters of dark matter halos on the base of dwarf galaxies' dynamics (Messier 32 and Leo I). For doing this, we propose the new approach founded on construction the coupled elliptical trajectory for a probe body in the gravitational fields of Newtonian potential and potential of dark matter's halo. This allows more accuracy estimate its central density for the Navarro-Frenk-White profile $\left(\rho_{0} \sim 10^{-24} \frac{\mathrm{g}}{\mathrm{cm}^{3}}\right)$ and free parameter for the Einasto profile $(0.06 \leq \alpha \leq 0.65)$. Our result is in good correlation with results of other authors that are got by different numerical methods.

\section{Keywords}

Dwarf Galaxies, Dark Matter Halo, Central Density of Dark Matter Profile, Parameters of Dark Matter's Halo

\section{Introduction}

One of the urgent problems of modern cosmology is searching the dark matter properties. Dark matter-an unusual type of cosmic substance, which is in the overall energy balance of the Universe approximately equals $27 \%$ [1]. Astronomical observations suggest that dark matter is concentrated around the large space objects such as galaxies and their clusters. Dark matter forms a halo which mass enriches about $90 \%$ of the galaxy total mass [2]. (Note, that dark matter may form different types of clumps without existence of any baryonic objects [1]). 
It should be noted that distribution of dark matter in the halo of galaxies is not uniform-it concentrates in their centers and decreases to peripheries. The corresponding distribution function of dark matter (profile) is usually founded on numerical methods that are modeling the dynamics of stars in galaxies. Today a number of profiles are known [3]-[7], which include the unknown dark matter density in the centers of galaxies $\rho_{0}$, as well as a number of free parameters.

Finding numerical values of these quantities is one of unsolved cosmological problems that from our viewpoint, can be partially solved by examining the dynamics of dwarf galaxies. Note that influence of dark energy and dark matter on galaxies' dynamics successfully described even in the framework of Newtonian approach [2] [8] [9]. Therefore, we'll be used it in our searching for estimation the magnitudes of the dark matter halo's parameters.

\section{On the Dynamics of Test Bodies in the Dark Matter Halo's of Massive Galaxies}

Now consider the following dynamic model -in the gravitational field of a massive galaxy, surrounded by a halo of dark matter, the probe body moves. The prototype of this model, for example, is the model of dwarf galaxy Messier 32 motion in the Andromeda galaxy or the motion of dwarf galaxy Leo I in the Milky Way. As for the distribution function of dark matter we'll choose two most known of them-Navarro-Frenk-White profile and Einasto profile.

\subsection{Navarro-Frenk-White Profile of Dark Matter}

The Navarro-Frenk-White profile looks as follows [10]

$$
\rho(r)=\frac{\rho_{0}}{\frac{r}{r_{0}}\left[1+\left(\frac{r}{r_{0}}\right)\right]^{2}} .
$$

Here and hereinafter $\rho_{0}$-the density of dark matter in the center of galaxy, $r_{0}$-the size of its halo, $r-$ the current radius. Assume that following relationship $\varepsilon=\frac{r}{r_{0}} \ll 1$ exists between them. Therefore, the profile function can be expanded in Taylor series with respect to above introduced small parameter. With the first order on $\varepsilon$ accuracy we get

$$
\rho(r)=\rho_{0}\left(\frac{r_{0}}{r}-2\right)
$$

Then the potential energy of the dark matter of the field can be written down as

$$
U=A r^{2}-B r,
$$

where coefficients in standard designations are

$$
A=\frac{8}{3} \pi G \rho_{0} m, B=\frac{4}{3} \pi G \rho_{0} m r_{0} .
$$

Later on we'll use first term in (3) only because such potential energy leads to a closed trajectory that corresponds to the real observations of dwarf galaxies' movement. Consequently, based on the conservation laws of energy and momentum for the test body dynamics, its trajectory can be write down in the standard form

$$
\begin{gathered}
\frac{\hat{p}}{r}=(1+\hat{e} \cos \psi)^{\frac{1}{2}} . \\
\psi=\arccos \frac{\left(\frac{1}{r^{2}}-\frac{m E}{M^{2}}\right)}{\sqrt{\frac{m^{2} E^{2}}{M^{4}}-2 \frac{m A}{M^{2}}}}+\text { const. }
\end{gathered}
$$




$$
\hat{p}^{2}=\frac{M^{2}}{m E}, \hat{e}=\sqrt{1-2 \frac{A M^{2}}{m E^{2}}} .
$$

From expressions (5)-(7) sees that it describes the elliptical trajectory that is similar to standard trajectory for one body in Newtonian mechanics [11]

$$
\begin{gathered}
\frac{p}{r}=1+e \cos \varphi, \\
\varphi=\arccos \frac{\frac{M}{r}-\frac{m \gamma}{M}}{\sqrt{2 m E+\frac{m^{2} \gamma^{2}}{M^{2}}}}+\text { const, }
\end{gathered}
$$

where $\gamma=G M_{g r}$ - constant of Newtonian potential for a gravitating mass $M_{g r}$. Marking $e=\frac{\hat{e}}{2}$ and $\hat{p}=p$, it's clear that expressions (5) and (8) can be written down in the form of coupled elliptical trajectory

$$
\frac{p}{r}=1+e(\cos \varphi+\cos \psi) .
$$

Now require that trajectories (5) and (8) coincide each other at the real movement of probe body. To substantiate this declaration we use the graphs of rotation curves for the Newtonian movement and for the movement of body in the gravitation field of dark matter. It is known that for the first case such curve is the hyperbola, while for the second case - the quasi-logarithmic line (for example, see [12] [13]). Observations have shown that these curves cross at the critical point $r_{c} \approx 5 \mathrm{Kpc}$ and the corresponding mean velocities at it approximately equals $v_{c} \approx 250 \frac{\mathrm{km}}{\mathrm{s}}$. (Really, for the dwarf galaxy Messier 32 the observable velocities interval is $179 \frac{\mathrm{km}}{\mathrm{s}} \leq v_{c} \leq 226 \frac{\mathrm{km}}{\mathrm{s}}$ [14]; for the dwarf galaxy Leo I-279 $\frac{\mathrm{km}}{\mathrm{s}} \leq v_{c} \leq 287 \frac{\mathrm{km}}{\mathrm{s}}$ [15]). Because of that we'll use these values for estimation of unknown dark matter halo's parameters.

Here it's necessary to point out that later on we'll consider distances no larger than $r$. At these distances curved line in the gravitation field of dark matter approximately looks like the straight line type of $v \sim r$. Note that such relation also follows from the equality centrifugal force and gravitational force of dark matter that relates to the first term in potential energy (3).

Put that angles of trajectories are coincide also, i.e. $\psi=\varphi$. Hence, the following equality takes place

$$
\frac{\frac{1}{r}\left(\frac{M}{r}-\frac{m E}{M} r\right)}{\sqrt{\frac{m^{2} E^{2}}{M^{2}}}-2 m A}=\frac{\frac{M}{r}-\frac{m \gamma}{M}}{\sqrt{2 m E+\frac{m^{2} \gamma^{2}}{M^{2}}}} .
$$

Since the total energy is larger than kinetic one and it, in its turn, is greater than potential energy

$(E>T>U)$, we can assume that $\frac{M}{r} \gg \frac{m \gamma}{M}$. Then expression (11) simplifies-

$$
\frac{\frac{1}{r}\left(\frac{M}{r}-\frac{m E}{M} r\right)}{\sqrt{\frac{m^{2} E^{2}}{M^{2}}-2 m A}}=\frac{\frac{M}{r}}{\sqrt{2 m E}} .
$$

Now from (12) can be found the value of an unknown factor in the first term of expression (3)

$$
A=-\frac{2 E^{3} m^{2} r^{2}-3 E^{2} m M^{2}}{2 M^{4}} .
$$


Comparison (4) and (13) allows get the expression of central density of dark matter's halos

$$
\rho_{0}=-\frac{3 E^{2}\left(2 E m r^{2}-3 M^{2}\right)}{16 \pi G M^{4}} .
$$

For numerical estimations assume that the probe mass $m$ in (14) equals to one. Thus roughly put $M=m \mu \sim r_{c} v_{c}, E=m \varepsilon \sim v_{c}^{2}$. After substitution the above mentioned numerical values for the position of probe body and its velocity, we obtain the following estimate of the central density of dark matter's halos

$$
\rho_{0} \sim 2.3 \times 10^{-24} \frac{\mathrm{g}}{\mathrm{cm}^{3}}
$$

It is interesting to compare this result with the previously obtained similar values. For example, in [16] $\rho_{0} \sim 10^{-16} \frac{\mathrm{g}}{\mathrm{cm}^{3}}$. However, in [17] it was shown that the central part of dark matter density should not be larger than $\rho_{0} \sim 10^{-18} \frac{\mathrm{g}}{\mathrm{cm}^{3}}$. Furthermore, in [3] [18] [19] it is shown that the central density of dark matter $\rho_{0} \sim 10^{-24} \frac{\mathrm{g}}{\mathrm{cm}^{3}}$. Analysis of these estimates gives that the most acceptable central density of the dark matter's halo, probably, lays within the interval $\sim 10^{-24}\left(\frac{\mathrm{g}}{\mathrm{cm}^{3}}\right) \leq \rho_{0} \leq \sim 10^{-18}\left(\frac{\mathrm{g}}{\mathrm{cm}^{3}}\right)$.

\subsection{Einasto Profile for Dark Matter}

Note that Navarro-Frank-White profile is completely identified. Nevertheless, in literature are known profiles of dark matter that contain from one [20] up to several [4] free parameters. In our article we'll consider Einasto profile [20]

$$
\rho(r)=\rho_{0} \exp \left\{-\frac{2}{\alpha}\left[\left(\frac{r}{r_{0}}\right)^{\alpha}-1\right]\right\}
$$

with one unknown parameter $\alpha$. Here as usual $\rho_{0}$-the density of dark matter in the center of galaxy, $r_{0}$-the size of its halo, $r$-the current radius. Based on the previously adopted relationship between size of the halo and the current radius, the exponential function of Einasto profile may be expanded in Taylor series. Restricting ourselves by two terms only we get

$$
\exp \left[\left(\frac{r}{r_{0}}\right)^{\alpha}-1\right]^{-\frac{2}{\alpha}} \approx 1-\frac{2}{\alpha}\left[\left(\frac{r}{r_{0}}\right)^{\alpha}-1\right] .
$$

In doing this the new additional condition we have used here- the smallness of the power parameter $\alpha$. Then Einasto profile in the approximate form is written down as

$$
\rho(r)=\hat{\rho}_{0}-\frac{2 \rho_{0}}{\alpha}\left(\frac{r}{r_{0}}\right)^{\alpha},
$$

where

$$
\hat{\rho}_{0}=\rho_{0}\left(1+\frac{2}{\alpha}\right)
$$

The potential energy of dark matter field in this case is as follows

$$
U=\hat{A} r^{\alpha+2}-\hat{B} r^{2},
$$

where

$$
\hat{A}=\frac{8}{3 \alpha r_{0}} \pi G \rho_{0} m, \quad \hat{B}=\frac{4}{3} \pi G \hat{\rho}_{0} m
$$


Now for further calculations we use only the second term in (22), because it leads to a closed trajectory as before. Therefore, using the conservation laws of energy and momentum, its trajectory can be written as

$$
\begin{gathered}
\frac{\hat{p}}{r}=(1+\hat{e} \cos 2 \hat{\varphi})^{\frac{1}{2}} . \\
\psi=\arccos \frac{\left(\frac{1}{r^{2}}-\frac{m E}{M^{2}}\right)}{\sqrt{\frac{m^{2} E^{2}}{M^{4}}+2 \frac{m \hat{B}}{M^{2}}}}+\text { const. } \\
\hat{p}^{2}=\frac{M^{2}}{m E}, \hat{e}=\sqrt{1+2 \frac{\hat{B} M^{2}}{m E^{2}}} .
\end{gathered}
$$

From the expression (24) sees that it describes an elliptical trajectory that is similar to the standard trajectory in Newtonian mechanics. Repeating the previous arguments about the procedure of analyzing trajectories and their shapes, we find the relation

$$
\frac{\frac{1}{r}\left(\frac{M}{r}-\frac{m E}{M} r\right)}{\sqrt{2 m \hat{B}+\frac{m^{2} E^{2}}{M^{2}}}}=\frac{\frac{M}{r}}{\sqrt{2 m E}} .
$$

Hence, the expression of an unknown coefficient $\hat{B}$ is

$$
\hat{B}=\frac{2 E^{3} m^{2} r^{2}-5 E^{2} m M^{2}}{2 M^{4}} .
$$

From the comparison of expressions (21), (26) and usage (19) we get

$$
\rho_{0}\left(1+\frac{2}{\alpha}\right)=\frac{3 E^{2}\left(2 E m r^{2}-5 M^{2}\right)}{8 \pi G M^{4}} .
$$

Important to notice that expression (27), besides dynamic characteristics of a particle $E$ and $M$, contains the current radius $r$ and unknown parameter $\alpha$. Basing on our previous article [21], it is possible to estimate and this parameter.

We write down the expression of central density of dark matter's halo in the form

$$
\rho_{0} \approx X \cdot 10^{-24} \frac{\mathrm{g}}{\mathrm{cm}^{3}} .
$$

Here the coefficient $X$ has various numerical values which are known in literature. In fact, from [3] it follows that $X_{1} \approx 0.7$; from [18] $-X_{2} \approx 0.7$; from [19] $-X_{3} \approx 0.5$; from [21] $-X_{4} \approx 2.3$ (see also (15)). As for the central density of dark matter's halo in Einasto profile, we write down it as

$$
\hat{\rho}_{0} \approx Y \cdot 10^{-24} \frac{\mathrm{g}}{\mathrm{cm}^{3}},
$$

where $Y$ is unknown coefficient. Substituting the necessary numerical values into (27) we find-for the dwarf galaxy Messier $32 \quad Y_{1} \approx 9.4$, for the dwarf galaxy Leo $\mathrm{I}-Y_{2} \approx 18.0$.

From comparison of (27), (28) and (29) follows the expression of unknown parameter $\alpha$

$$
\alpha=2 /\left(\frac{Y}{X}-1\right)
$$

Using the found values $X$ and $Y$ we can obtain this parameter. As for the galaxy Messier $32 \quad Y_{1} \approx 9.4$ and above given values of $X$, we find: $\alpha_{1} \approx \alpha_{2} \approx 0.16 ; \alpha_{3} \approx 0.11 ; \alpha_{4} \approx 0.65$. And finally, for the Galaxy Leo I when $Y_{2} \approx 18.0$ we have: $\alpha_{1} \approx \alpha_{2} \approx 0.08 ; \alpha_{3} \approx 0.06 ; \alpha_{4} \approx 0.40$. (At calculations we took into account that for such galaxy $X_{4} \approx 3.0$ ). 
From the saying above we conclude-free parameter in Einasto profile must satisfy the following interval

$$
0.06 \leq \alpha \leq 0.65 \text {. }
$$

Note that in article [1] the corresponding interval is closed to (31) and equals $0.16 \leq \alpha \leq 0.30$. Also important to emphasize that in [22] the next interval of parameter $\alpha$ have been argued $0.39 \leq \alpha \leq 15.0$.

\section{Conclusions}

In the article, the new method for search characteristics of dark matter's halo is proposed. Searching the Navarro-Frenk-White profile shows that the central part of density of dark matter's halos must satisfy the following magnitude $\rho_{0} \sim 10^{-24}\left(\frac{\mathrm{g}}{\mathrm{cm}^{3}}\right)$. This estimation allowed find the interval for free parameter of Einasto profile $0.06 \leq \alpha \leq 0.65$. Remarkable that it is close to similar intervals given in recent articles [22]-[24], especially in [1]. That is why expedient to compare few methods of the Einasto parameter finding.

Authors of [22] used the semidegenerate thermal self-gravitating general relativistic fermionic gas as the dark matter model. As the result they got the dark matter profile in the form of broken line of four regions. First of them describes by rotation curve type of $v \sim r$ (the correctness of which on small distances we point out before), fourth of them — by the quasi-logarithmic line (that is correct for the large space scales). The peculiarity of such profile consists in existence of two intermediate regions for the moderate distances.

For describing this broken line in terms of Einasto profile (and not only) they used results of the survey THINGS were have been obtained highest quality rotational curves for 34 nearby spiral and irregular galaxies. Naturally that these curves depend not only the dark matter, but on some baryonic parts (baryonic substrate, relativistic gas, etc.) and their quantitative relation also. Therefore authors got the cited above range of Einasto parameter $0.39 \leq \alpha \leq 15.0$.

In the framework of N-body simulations- “Aquarius” project with number of particles is 4.4 billions-authors [23] used Einasto profile in the form $\rho(r) \sim r^{\alpha}$. Approximately their result is $0.13 \leq \alpha \leq 0.17$ and looks like the quasi-permanent parameter. Nevertheless they pointed out that the magnitude of Einasto parameter slightly varies from halo to halo because different halos cannot, in general, be identical. Therefore power parameter must have the more width numerical interval.

Really, in [24] in the framework of "Aquarius" project also, but for one order smaller number of particles, was shown that Einasto parameter lays within the interval $0.12 \leq \alpha \leq 0.35$. In doing this authors emphasize that this parameter changes together with growth of galaxy's mass. They also point out that if the shape of Einasto profile tends to isotherml one $\alpha=0$, while for Gaussian profile $\alpha=2$. So, the sought-for interval in reality must be $0 \leq \alpha \leq 2.0$.

From this examination, it's clear the difference between ours models-in our case model based on the dwarf galaxies Newtonian dynamics and allowed get the improved estimate of dark matter core. Moreover, it's very simple and allowed get the very plausible interval for the Einasto parameter. In contrary, the model in [22] is relativistic one, it's based on the precisian curve lines observations but have the essentially width interval for Einasto parameter.

Two above mentioned other articles [23] [24], as sees, are based on the N-body simulations method and give the closed to our power parameter $\alpha$. Together with that these articles devoted to study other unsolved problems - mass, velocity dispersion and anisotropy profiles of cold dark matter halos and its evolution, while they are not been under our consideration.

Therefore all of these models must be regard as additional each other which give rather satisfactory description of dark matter halo on different cosmological scales and for different space objects.

\section{References}

[1] Berezinsky, B.S., Dokuchaev, B.I. and Eroshenko, Y.N. (2014) Advances in Physical Sciences, 184, 3-42. (In Russian)

[2] Dolgachev, V.P., Domozhilova, L.M. and Chernin, A.D. (2003) Astronomy Reports, 47, 728-732.

[3] Iocco, F., Pato, M., Bertone, G. and Jetzer, P. (2011) Journal of Cosmology and Astroparticle Physics, 11, 29.

[4] Avila-Reese, V., Firmani, C., Klypin, A. and Kravtsov, A.V. (1999) Monthly Notices of the Royal Astronomical Society, 310, 527-539.

[5] Burkert, A. (1995) The Astrophysical Journal Letters, 447, 171-175. 
[6] Catena, R. and Ullio, P. (2010) Journal of Cosmology and Astroparticle Physics, 8, 1-21.

[7] Evans, N.W. and An, J. (2006) Physical Review D, 73, Article ID: 023524.

[8] Kahn, F.D. and Woljter, L. (1959) The Astrophysical Journal, 130, 705-717.

[9] Chechin, L.M. and Ibraimova, A.T. (2015) Astronomical and Astrophysical Transactions, in Press.

[10] Navarro, J.F., Frenk, C.S. and White, S.D.M. (1996) The Astrophysical Journal, 462, 563.

[11] Landau, L.D. and Lifschitz, E.M. (1988) Mechanics. M. Nauka.

[12] Kirilov, A.A. and Turaev, D. (2006) Monthly Notices of the Royal Astronomical Society, 371, L31-L35.

[13] Kravtsov, A.V. and Klypin, A.A. (1998) The Astrophysical Journal, 502, 48.

[14] Howley, K.M., Guhathakurta, P., van der Marel, R., Geha, M., Kalirai, J., Yniguez, B., Kirby, E., Cuillandre, J.-C. and Gilbert, K. (2013) The Astrophysical Journal, 765, 22.

[15] Lokas, E.L., Klimentowski, J., Kazantzidis, S. and Mayer, L. (2008) Monthly Notices of the Royal Astronomical Society, 390, 625-634.

[16] Hideyoshi, A. (2010) Advances in Space Research, 45, 1007-1014.

[17] Chechin, L.M. (2013) International Journal of Astronomy and Astrophysics, 3, 285-290.

[18] Nesti, F. and Salucci, P. (2012) The Local Dark Matter Density. VIII International Workshop on the Dark Side of the Universe, Rio de Janeiro, 10-15 June 2012.

[19] Zacek, V. (2007) Dark Matter. Fundamental Interactions: Proceedings of the 22nd Lake Louise Winter Institute, World Scientific Publishing Co. Pte. Ltd., 170-206.

[20] Einasto, J. (2000) Astronomical Soc. Pacific Conference Series, 252, 85.

[21] Chechin, L.M. and Konysbayev, T.K. (2016) Reports of the National Academy of Sciences of Kazakhstan. (In Russian, in Press)

[22] Siutsou, I., Argüelles, C.R. and Ruffini, R. (2015) Astronomy Reports, 59, 656-666.

[23] Navarro, J.F., Ludlow, A., Springel, V., et al. (2009) MNRAS, 402, 21-24.

[24] Dutton, A.A. and Andrea Macciò, V. (2014) MNRAS, 441, 3359-3374. 